\title{
Erratum to: Improved Generation of Higher Harmonics and Suppression of the Lowest Harmonics in an X-Ray FEL with a Two-Frequency Undulator
}

\author{
K. V. Zhukovsky \\ Moscow State University, Moscow, 119991 Russia \\ Received April 08, 2019; revised April 08, 2019; accepted April 08, 2019
}

DOI: $10.3103 / \mathrm{S} 0027134919030111$

We hereby clarify the following correct mathematical form of formula (5) and formulas in the text above it for the Bessel coefficients of a dual-frequency undulator:

$$
\begin{gathered}
f_{n, x}=I_{n-1}^{(h)}(n)+I_{n+1}^{(h)}(n) \\
=\frac{d}{h}\left(I_{n+h}^{(h)}(n)+I_{n-h}^{(h)}(n)\right), \\
I_{n}^{(h)}(m)=\int_{0}^{2 \pi} \frac{d \phi}{2 \pi} \\
\times \cos \left(n \phi+\frac{m k^{2}\left(\xi_{1}+\xi_{2}+\xi_{3}+\xi_{4}\right)}{1+k_{\mathrm{eff}}^{2} / 2}\right), \\
\xi_{1}=\frac{\sin (2 \phi)}{4}, \quad \xi_{2}=\frac{d \sin ((h-1) \phi)}{h(h-1)}, \\
\xi_{3}=\frac{d \sin ((h+1) \phi)}{h(h+1)}, \quad \xi_{4}=\frac{d^{2} \sin (2 h \phi)}{4 h^{3}} .
\end{gathered}
$$

E-mail: zhukovsk@physics.msu.ru

The original article can be found online at https://doi.org/10.3103/S0027134918050193. 